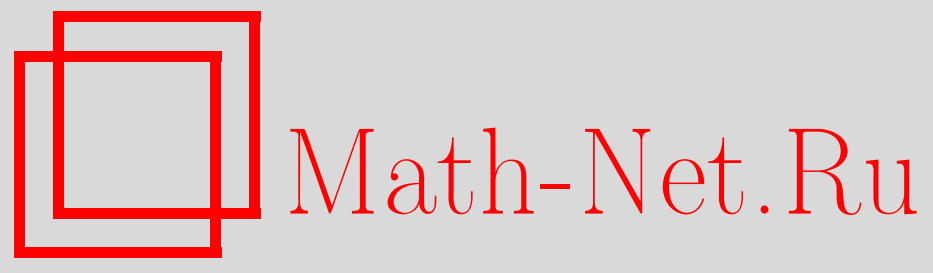

Н. М. Комарцов, М. А. Кулагина, Б. А. Рычков, О трактовке модуля упругости горных пород, Вестн. Сам. гос. техн. ун-та. Сер. Физ.-мат. науки, 2018, номер 3, 487-503

DOI: https://doi.org/10.14498/vsgtu1604

Использование Общероссийского математического портала MathNet.Ru подразумевает, что вы прочитали и согласны с пользовательским соглашением

http://www.mathnet.ru/rus/agreement

Параметры загрузки:

IP : 54.81 .137 .203

26 апреля 2023 г., 11:23:36

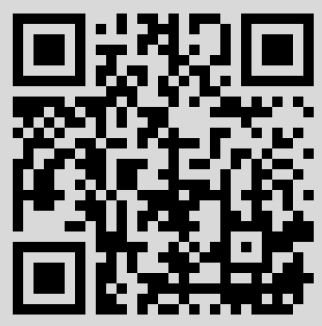


УДК 539.32:624.13

\title{
О трактовке модуля упругости горных пород
}

\author{
Н. М. Комарцов, М. А. Кулагина, Б. А. Ръчков \\ Кыргызско-Российский Славянский университет им. Б. Н. Ельцина, \\ Кыргызская Республика, 720000, Бишкек, ул. Киевская, 44.
}

\section{Аннотация}

Рассмотрены экспериментальные данные для горных пород, представленные в монографии K. Mogi "Experimental rock mechanics", изданной в 2007 году. Цилиндрические образцы пород были испытаны по схеме Т. Кармана: вначале создавалось гидростатическое давление до разного уровня напряжений, после чего увеличивалась осевая нагрузка при постоянном достигнутом боковом давлении. При таком сложном нагружении измерялось (на втором этапе нагружения) только приращение осевой деформации в зависимости от приращения осевого напряжения. Эта зависимость (в оригинале) представлена в виде графиков в реальном масштабе, что дало возможность (в настоящей работе) перевести данные графики в цифровой формат в виде табличных значений. Рассмотрены две горные породы: диорит (Orikabe Diorite) и перидотит (Nabe-ishi Peridotite).

По полученным таблицам проанализировано напряженно-деформированное состояние указанных пород на втором этапе сложного нагружения для шести осуществленных в опыте программ испытания. На каждой (из шести) реализованных траекторий нагружения выделена такая точка, которая соответствует осевому напряжению при одном и том же виде напряженного состояния. Последний, как это принято в геомеханике, характеризуется отношением среднего главного напряжения к максимальному главному напряжению. Таким образом выделяется (расчетная) траектория пропорционального нагружения для всех уровней напряжений в пределах упругости. Продемонстрировано, что для таких расчетных траекторий нагружения экспериментальное значение приращения осевой деформации (в пределах упругости) является линей-

\section{Научная статья}

(2)(1) Контент публикуется на условиях лицензии Creative Commons Attribution 4.0 International (https://creativecommons.org/licenses/by/4.0/deed.ru)

\section{Образец для цитирования}

Комарцов Н. М., Кулагина М. А., Рычков Б. А. О трактовке модуля упругости горных пород // Вестн. Сам. гос. техн. ун-та. Сер. Физ.-мат. науки, 2018. Т. 22, № 3. C. 487-503. doi: $10.14498 /$ vsgtu1604.

\section{Сведения об авторах}

Никита Михайлович Комарцов (1) http://orcid.org/0000-0003-0266-6271 кандидат физико-математических наук, доцент; e-mail: komartsovnm@mail.ru Маргарита Алексеевна Кулагина (1) http://orcid.org/0000-0001-7979-7881 аспирант; e-mail: kulagina_m.a@mail.ru

Борис Александрович Рычков (10) http://orcid.org/0000-0001-7408-1657 доктор физико-математических наук, професcop; e-mail: rychkovba@mail.ru 
ной функцией от приращения осевого напряжения. Этим доказывается применимость обобщенного закона Гука.

В итоге определены модуль Юнга и коэффициент Пуассона и показано, что они (применительно и к горным породам) действительно являются упругими константами, а не переменными величинами, как это иногда трактуется.

Ключевые слова: упругая деформация горных пород, упругие константы, применимость закона Гука при сложном нагружении.

Получение: 30 января 2018 г. / Исправление: 17 августа 2018 г. / Принятие: 3 сентября 2018 г. / Публикация онлайн: 9 октября 2018 г.

Введение. Горные породы на определенной глубине в массиве находятся в полуразрушенном состоянии под действием неравномерных объемных давлений. Такое состояние в лабораторных условиях моделируется неравномерным трехосным сжатием цилиндрических образцов горных пород. При этом получают так называемую полную диаграмму «напряжение-деформация». «Восходящая» ветвь этой диаграммы отражает деформацию до предела прочности (напряжение постоянно растёт); затем следует «нисходящая» ветвь, когда деформация растёт (при понижении напряжения), но сохраняется несущая способность образца вплоть до предела остаточной прочности, при достижении которой образец разделяется на отдельные части. В результате таких испытаний образцов разнообразных пород определяются их механические свойства [1-4], которые затем используются для решения прикладных задач при разработке полезных ископаемых. В данной работе рассмотрена восходящая ветвь указанной полной диаграммы до условного предела упругости; использованы экспериментальные данные, представленные в монографии [1], в частности, для диорита (Orikabe Diorite) и перидотита (Nabe-ishi Peridotite).

Одним из наиболее распространенных видов испытаний образцов горных пород является испытание по схеме Т. Кармана, когда вначале создается гидростатическое давление цилиндрического образца, а затем при постоянном боковом давлении увеличивается осевое давление. В этом случае сложного нагружения (по двузвенной ломаной в пространстве напряжений) обычно фиксируется только приращение осевой деформации, вызванное приращением осевой нагрузки (соответствующей второму звену траектории нагружения). Это обстоятельство приводит к затруднению при определении упругих свойств материала, характеризуемых обобщенным законом Гука. На самом деле подобные затруднения возникают даже при определении модуля Юнга при одноосном сжатии. Считается [5], что ГОСТ 28985-97 [6] в настоящее время устарел, т.к. он не учитывает современного развития экспериментальной техники. Определенные недостатки отмечаются также [5] у стандартов DIN EN 14580:2005-07 [7] и ASTM D7012-10 [8]. Техника проведения соответствующих экспериментов постоянно совершенствуется [9-15]. Кроме того, осу- 
ществляются эксперименты по определению упругих свойств горных пород при циклическом нагружении, некоторые из них описаны в работах $[16,17]$.

В данном сообщении основное внимание уделяется трактовке модуля упругости как действительной константы материала для горных пород на основе проанализированных (указанных выше) испытаний на сложное нагружение.

1. Исходные данные. На представленных в монографии [1] графиках отражены приращения осевой деформации при соответствующем приращении осевого напряжения и постоянном достигнутом равномерном боковом давлении после начального гидростатического давления образца до определенной величины. Экспериментальные данные для диорита (Orikabe Diorite, рис. 1) и перидотита (Nabe-ishi Peridotite, рис. 2) воспроизведены с оригинала [1]. Как было проверено автором эксперимента, в исходном состоянии эти горные породы представляют собой изотропный материал. Была проведена оцифровка этих графиков, а полученный результат использован в виде табличных значений деформаций и напряжений.

На рис. 1 и 2 приращение осевой деформации обозначено $\varepsilon$, а разность $\left(\sigma_{1}-\sigma_{3}\right)$ фактически означает приращение осевого напряжения, поскольку после гидростатического давления (до уровня $P_{H}$ ) осевое напряжение $\sigma_{1}>\sigma_{2}=\sigma_{3}=$ const.

2. Определение упругих констант. Как известно, для определения упругих констант начально изотропного материала правомерно применять обобщенный закон Гука. В частности, в рассматриваемом случае для осевой деформации имеем

$$
\varepsilon_{1}=\frac{1}{E}\left[\sigma_{1}-\nu\left(\sigma_{2}+\sigma_{3}\right)\right]
$$

где $E$ - модуль Юнга, $\nu$ - коэффициент Пуассона.

Согласно основному постулату теории упругости, траектория рассматриваемого сложного нагружения в конечной ее точке заменяется пропорциональным нагружением в эту точку, что, по определению, доставляет ту же самую величину конечной деформации, как и при сложном нагружении. Если вид напряженного состояния характеризовать параметром $c=$ $=\sigma_{2} / \sigma_{1}\left(\sigma_{1}\right.$ - осевое напряжение, $\sigma_{2}=\sigma_{3}$-напряжения от бокового давления, $\left.\sigma_{1}>\sigma_{2}=\sigma_{3}\right)$, то формулу (1) можно представить в виде

$$
\varepsilon_{1}=\frac{\sigma_{1}}{E}[1-2 c \nu]
$$

При одном и том же виде напряженного состояния $(c=$ const), т.е. при пропорциональном нагружении, формула (2) справедлива и для приращений напряжений и деформаций:

$$
\Delta \varepsilon_{1}=\frac{\Delta \sigma_{1}}{E}[1-2 c \nu], \quad c=\text { const. }
$$

Выбирая значения исходных (зафиксированных в опыте, например, для диорита) приращений напряжений и деформаций при нескольких значениях 


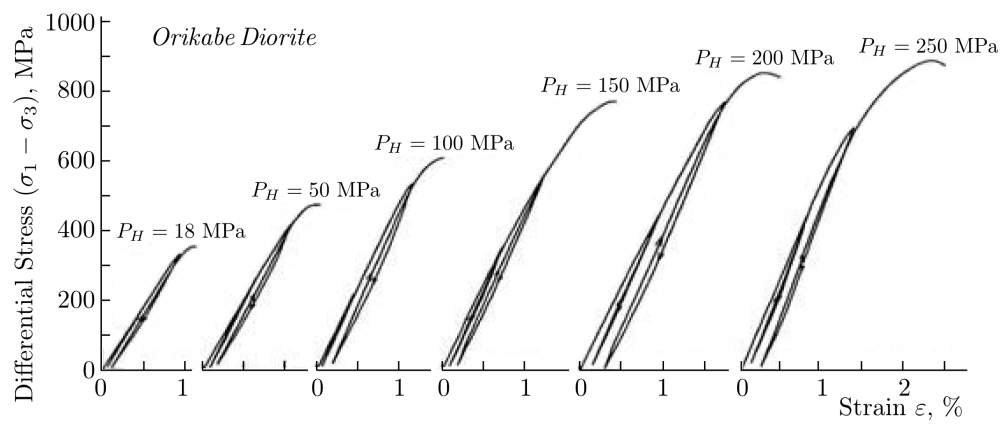

Рис. 1. Экспериментальные зависимости «приращение осевого напряжения-приращение осевой деформации» для диорита (воспроизведено с [1])

[Figure 1. Experimental dependences of the increment of the axial stress-increments of axial strain for Orikabe Diorite (reproduced from [1])]

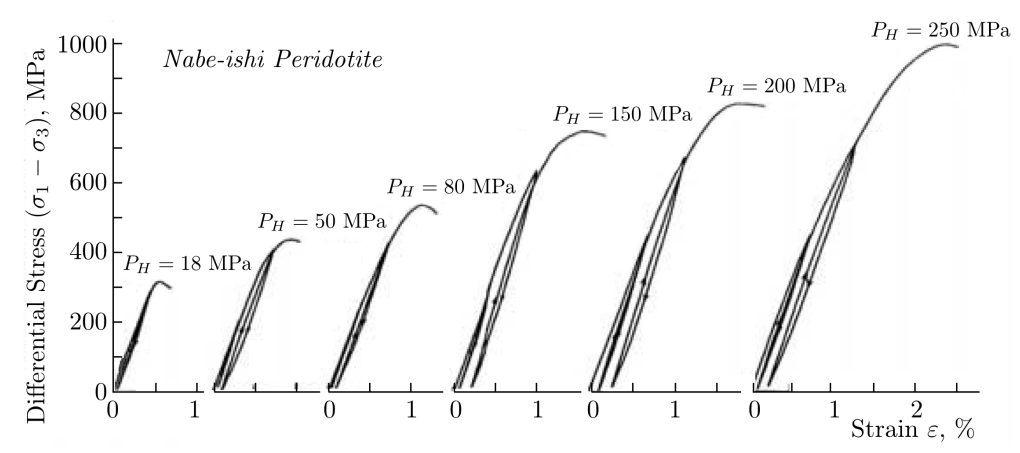

Рис. 2. Экспериментальные зависимости «приращение осевого напряжения-приращение осевой деформации» для перидотита (воспроизведено с [1])

[Figure 2. [Experimental dependences of the increment of the axial stress-increments of axial strain for Nabe-ishi Peridotite (reproduced from [1])]

параметра $c$ для всех осуществленных давлений, действительно получили соответствующие линейные зависимости $\Delta \varepsilon_{1}\left(\Delta \sigma_{1}\right)$, представленные на рис. 3 до условного предела упругости.

При фиксированном боковом давлении диаграмма $\Delta \varepsilon_{1} \sim \Delta \sigma_{1}$ является нелинейной в пределах упругости, поскольку с изменением осевого напряжения соответственно изменяется и вид напряженного состояния $(c)$. Для подобных диаграмм деформаций предел упругости $\sigma_{1 e}$ определяется согласно методике [18], по которой тангенс угла наклона касательной к кривой деформации в начале координат и в точке, соответствующей пределу упругости, может изменяться до $50 \%$. Применительно к рассматриваемым горным породам указанное изменение углового коэффициента касательной применялось в пределах 20-25\%. Расчетные значения пределов упругости приведены в табл. 1.

Рассматривая попарно диаграммы $\Delta \varepsilon_{1} \sim \Delta \sigma_{1}$ при двух из выбранных значений параметра $c\left(c_{1}\right.$ и $\left.c_{2}\right)$, определим согласно зависимости $(3)$ модуль Юнга $E$ и коэффициент Пуассона $\nu$. Полученные при разных комбинациях 


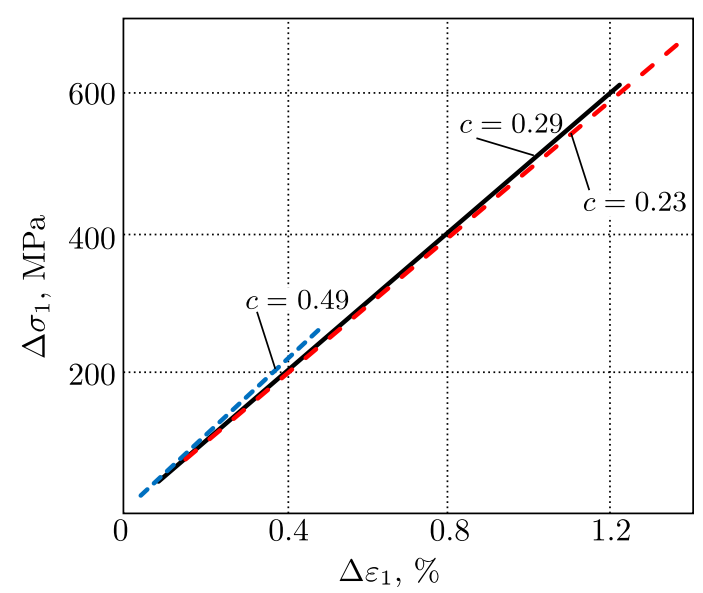

Рис. 3. Графики зависимости $\Delta \varepsilon_{1}\left(\Delta \sigma_{1}\right)$ для диорита при различных значениях $c$

[Figure 3. Plots of dependence between $\Delta \varepsilon_{1}$ and $\Delta \sigma_{1}$ for Orikabe Diorite, when the values of $c$ are various]

параметров $c_{1}$ и $c_{2}$ значения величин $E$ и $\nu$ затем усредняются, что представлено в табл. 2 для диорита, в которой отклонения $\Delta E$ и $\Delta \nu$ от средних значений $E_{a v}$ и $\nu_{a v}$ определялись по формулам

$$
\Delta E=\frac{E-E_{a v}}{E_{a v}} \cdot 100 \%, \quad \Delta \nu=\frac{\nu-\nu_{a v}}{\nu_{a v}} \cdot 100 \% .
$$

Как следует из табл. 2, неизбежно возникает расхождение (в виде отклонений (4)) между усредненными величинами и соответствующими расчетными значениями определяемых параметров при конкретных видах напряженного состояния. Это обстоятельство является следствием незначительной разницы в характере упрочнения породы при сопоставлении диаграмм $\Delta \varepsilon_{1} \sim \Delta \sigma_{1}$ при разных с. Эта картина для перидотита еще более усугубляется - при

Таблица 1 Значения пределов упругости $\sigma_{1 e}$ в МПа [The values of the elasticity limits $\sigma_{1 e}, \mathrm{MPa}$ ]

\begin{tabular}{l|c|c|c|c}
\hline \multirow{2}{*}{ Rock } & \multicolumn{4}{|c}{ Pressure $P_{H}, \mathrm{MPa}$} \\
\cline { 2 - 5 } & 50 & 150 & 200 & 250 \\
\hline Orikabe Diorite & 433 & 649 & 786 & 822 \\
Nabe-ishi Peridotite & 420 & 636 & 670 & 795 \\
\hline
\end{tabular}

Таблица 2 Упругие константы $\nu$ и $E$ для диорита [Elastic constants $\nu$ and $E$ for Orikabe Diorite]

\begin{tabular}{c|c|c|c|r|r}
\hline$c_{1}$ & $c_{2}$ & $\nu$ & $E, \mathrm{MPa}$ & \multicolumn{1}{c|}{$\Delta \nu, \%$} & \multicolumn{1}{c}{$\Delta E, \%$} \\
\hline 0.29 & 0.49 & 0.171693 & 44851.623 & 5.68215 & -0.67603 \\
0.49 & 0.23 & 0.166863 & 45106.891 & 2.70891 & -0.11074 \\
0.29 & 0.23 & 0.148829 & 45512.174 & -8.39106 & 0.78676 \\
\hline \multicolumn{2}{l}{ Average value } & 0.162462 & 45156.896 & \multicolumn{3}{l}{} \\
\hline
\end{tabular}


некоторых значениях $c$ диаграммы $\Delta \varepsilon_{1} \sim \Delta \sigma_{1}$ трудноразличимы (практически сливаются). Поэтому для этой горной породы были выбраны такие диаграммы $\Delta \varepsilon_{1} \sim \Delta \sigma_{1}$ при двух значениях $c\left(c_{1}=0.25\right.$ и $\left.c_{2}=0.42\right)$, которые являются наиболее контрастными, если их сравнивать по уровню упрочнения. В результате для перидотита получены следующие константы упругости: $E=54474.74$ МПа и $\nu=0.249$.

3. Анализ определения упругих параметров горной породы по экспериментальным данным сложного нагружения. В монографии [1] на втором звене рассматриваемой траектории сложного нагружения введен только один параметр $\left(E_{f}\right)$, характеризующий упругую деформацию. Утверждается, что

$$
\Delta \varepsilon_{1}=\varepsilon_{1}-\varepsilon_{1}^{*}=\frac{\sigma_{1}-\sigma_{1}^{*}}{E_{f}} .
$$

Зависимость (5) формально получается, если от текущего значения осевой деформации $\varepsilon_{1}$ вычесть ее значение $\varepsilon_{1}^{*}$, достигнутое при предварительном гидростатическом давлении (при $\sigma_{1}^{*}=\sigma_{2}^{*}=\sigma_{3}^{*}$ ). В этом случае деформации $\varepsilon_{1}$ и $\varepsilon_{1}^{*}$ теоретически вычисляются по формуле (1). Поскольку $\sigma_{2}^{*}=\sigma_{3}^{*}=$ const, в получаемой таким образом формуле (5) для приращения осевой деформации $\Delta \varepsilon_{1}$ теряется влияние (на ее величину) коэффициента Пуассона. Фактически при этом сравниваются деформации для разных траекторий пропорционального нагружения, что противоречит указанному выше основному постулату теории упругости. Поэтому вычисление $\Delta \varepsilon_{1}$ следует производить по формуле (2). В отличие от первоисточника, модуль $E_{f}$ в формуле (5) мы обозначили с нижним индексом, т.е. это на самом деле некоторый приведенный (или фиктивный) модуль. Если сравнить формулы (2) и (3), то указанный фиктивный модуль выражается через действительный модуль Юнга и коэффициент Пуассона:

$$
E_{f}=\frac{E}{1-2 c \nu}
$$

Из последней формулы видно, что модуль $E_{f}$ является переменной величиной, зависящей от вида напряженного состояния и от достигаемой упругой деформации. Действительно, в эксперименте получены следующие значения модуля $E_{f}$, отождествляемого автором [1] с модулем Юнга $E$, представленные (согласно оригиналу) на рис. 4 для всех боковых давлений. На этом рисунке в соответствии с условиями проведения эксперимента по оси абсцисс зафиксировано приращение осевой деформации для второго звена траектории нагружения, обозначенное через $\varepsilon$ (как в оригинале).

Подобная картина изменения (фиктивного) модуля упругости $E_{f}$ в аналогичных условиях испытаний приводится и в других исследованиях [3,4]. Исходные экспериментальные данные для дальнейшего анализа представлены на рис. 5 , где приращение осевого напряжения $\Delta \sigma_{1}$ откладывается от уровня гидростатического давления, достигнутого при разных боковых давлениях (18-250 МПа). На этом рисунке указаны также зависимости $\Delta \varepsilon_{1}\left(\Delta \sigma_{1}\right)$ при двух значениях вида напряженного состояния $c$, отражающих все осуществ- 

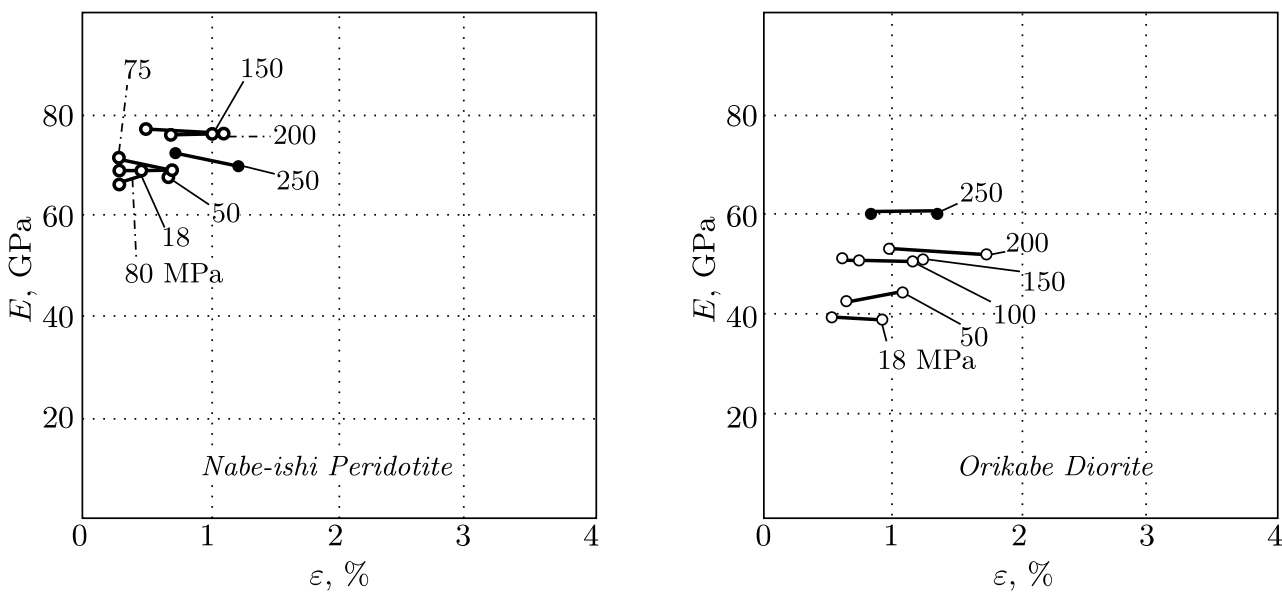

Рис. 4. Зависимость приведенного (фиктивного) модуля упругости от приращения осевой деформации для перидотита и диорита (воспроизведено с [1])

[Figure 4. Dependence of the reduced (fictitious) modulus of elasticity on the increment of axial deformation for Nabe-ishi Peridotite and Orikabe Diorite (reproduced from [1])]

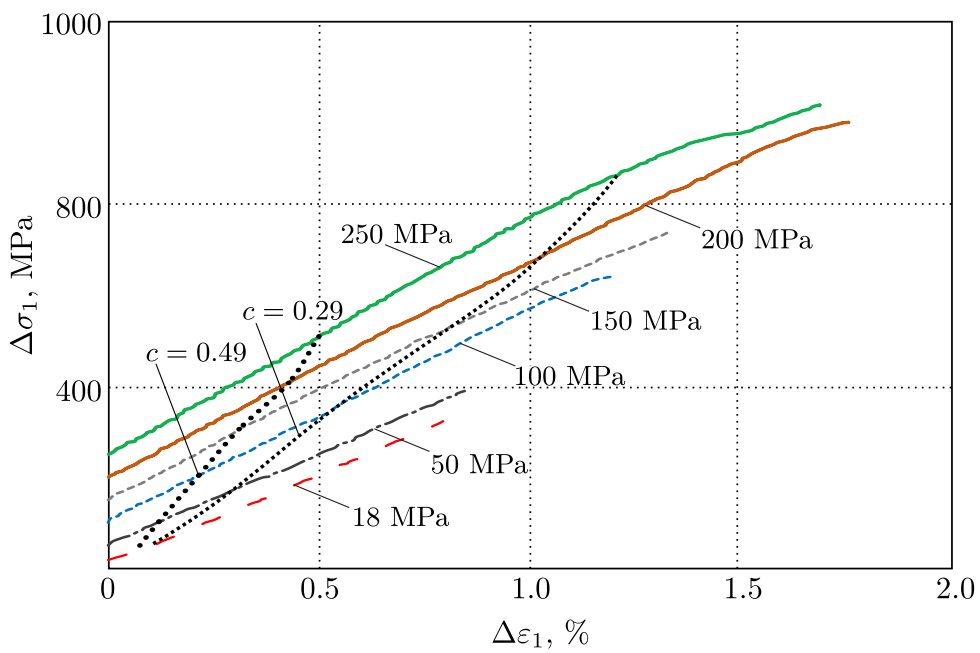

Рис. 5. Зависимости $\Delta \varepsilon_{1}\left(\Delta \sigma_{1}\right)$ для диорита вплоть до условного предела упругости при разных постоянных боковых давлениях

[Figure 5. Plots of dependence between $\Delta \varepsilon_{1}$ and $\Delta \sigma_{1}$ for Orikabe Diorite up to the conditional elastic limit at different constant lateral pressures]

ленные боковые давления. Эти данные можно использовать при вычислении величины $E_{f}$ согласно (6).

Сопоставление расчетных и экспериментальных данных относительно $E_{f}$ представлено на рис. 6.

Несмотря на отмеченные затруднения при определении упругих констант $E$ и $\nu$ по предлагаемому методу, получено доказательство того, что модуль Юнга $E$ действительно является константой материала. Это подтверждается, в частности, отображением реальной картины изменения параметра $E_{f}$ как некоторой эмпирической величины. 

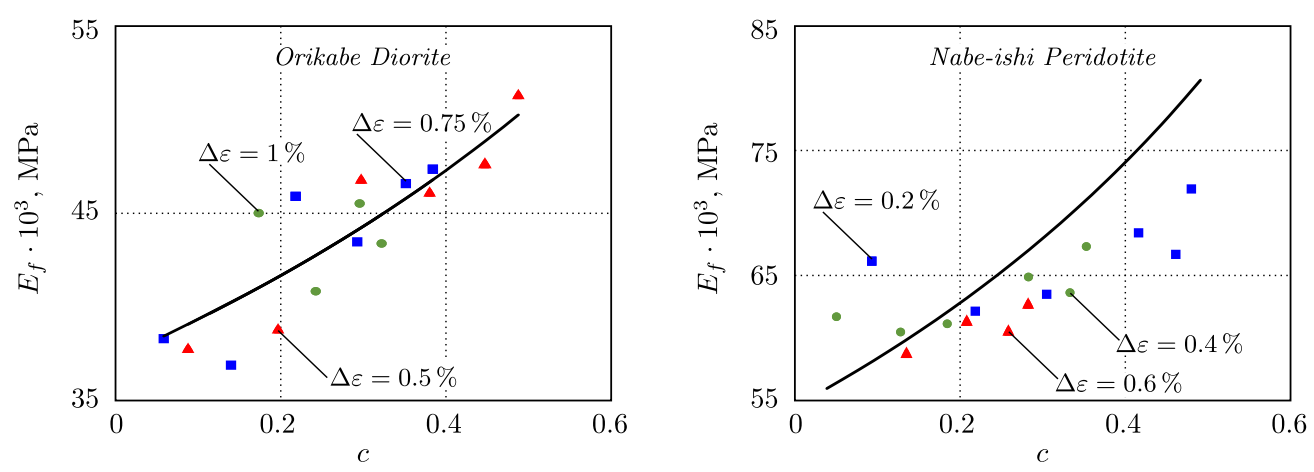

Рис. 6. Расчетные (сплошные линии) и экспериментальные (точки) значения фиктивного модуля упругости $E_{f}$ для диорита и перидотита

[Figure 6 The calculated (solid lines) and experimental (points) values of the fictitious modulus $E_{f}$ for Orikabe Diorite and Nabe-ishi Peridotite]

4. Возможная корректировка значений модуля Юнга и коэффициента Пуассона. Определение упругих констант можно еще осуществлять следующим образом. На втором звене траектории нагружения выделим какое-либо одно и то же приращение напряжения $\Delta \sigma_{1}$, достигаемое при разных боковых давлениях. Рассматривая зависимость $\Delta \varepsilon_{1}\left(\Delta \sigma_{1}\right)$ при различных комбинациях двух значений бокового давления, можно, как и по предыдущему методу, используя (3), вычислить константы $E$ и $\nu$. Для диорита получены константы $E=40660$ МПа и $\nu=0.23$, для перидотита $-E=56490$ МПа, $\nu=0.21$. Проверим, насколько лучше отражается общая картина деформирования при том или ином методе определения упругих констант. Для этого построим зависимость приращения объемной деформации

$$
\Delta \varepsilon=\frac{1}{3}\left(\Delta \varepsilon_{1}+\Delta \varepsilon_{2}+\Delta \varepsilon_{3}\right) \quad\left(\Delta \varepsilon_{2}=\Delta \varepsilon_{3}\right)
$$

от приращения среднего давления

$$
\Delta \sigma=\frac{1}{3} \Delta \sigma_{1}(1+2 c) \quad\left(c=\frac{\sigma_{2}}{\sigma_{1}}, \sigma_{2}=\sigma_{3}\right) .
$$

Приращение поперечной деформации представляется подобно формуле (2):

$$
\Delta \varepsilon_{2}=\frac{\Delta \sigma_{1}}{E}[c-\nu(1+c)]
$$

Согласно (3), (7), (8) и (9), имеем

$$
\Delta \varepsilon=\frac{1}{3} \frac{\Delta \sigma_{1}}{E}(1-2 \nu)(1+2 c) .
$$

Поскольку в эксперименте поперечная деформация не измерялась, при сравнении теоретических и экспериментальных данных значения $\Delta \varepsilon_{2}=\Delta \varepsilon_{3}$ во всех случаях вычислялись по формуле (10). Сопоставление теоретической 
и соответствующей «полуэмпирической» зависимости $\Delta \varepsilon(\Delta \sigma)$ для диорита представлено на рис. 7, а для перидотита - на рис. 8.

Из данных, представленных на рис. 7, 8, видно, что второй метод определения упругих констант предпочтительнее, поскольку в этом случае «полуэмпирическая» зависимость $\Delta \varepsilon(\Delta \sigma)$ лучше соответствует теоретической линейной связи между объемной деформацией и средним давлением. Оконча-
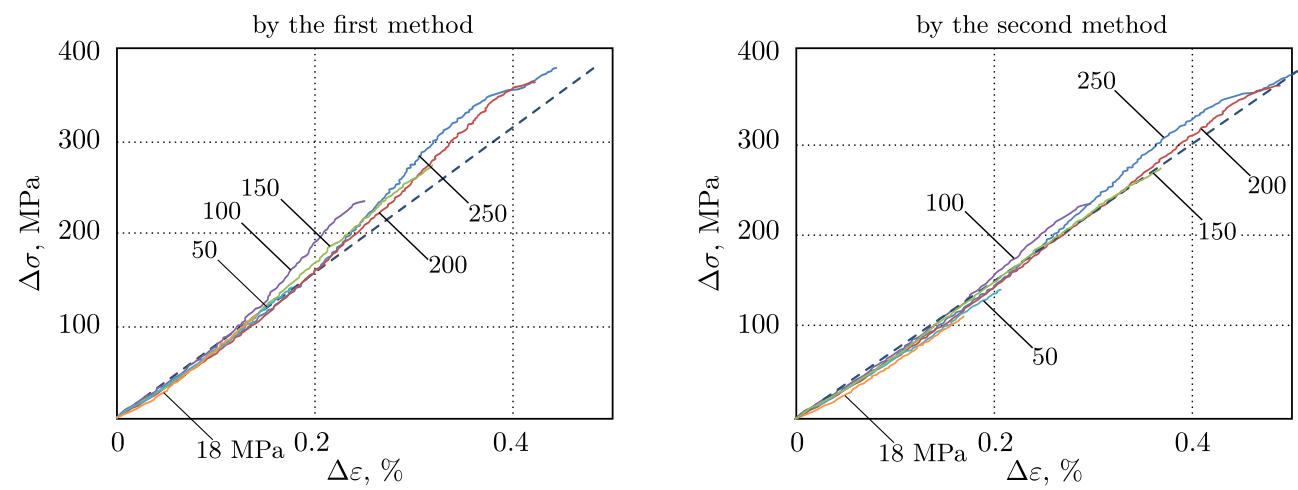

Рис. 7. (онлайн в цвете) Зависимость приращения объемной деформации $\Delta \varepsilon$ от приращения среднего давления $\Delta \sigma$ для диорита: штриховая линия-теоретическая зависимость, сплошные линии - зависимости для боковых давлений, когда упругие константы определены по первому способу (слева) и по второму способу (справа)

[Figure 7. (color online) Plots of dependence between a volume deformation increment $(\Delta \varepsilon)$ and a mean pressure $(\Delta \sigma)$ for Orikabe Diorite: dashed line is theoretical dependence, solid lines are dependencies for lateral pressures when the elastic constants are defined by the first method (left) and by the second method (right)]
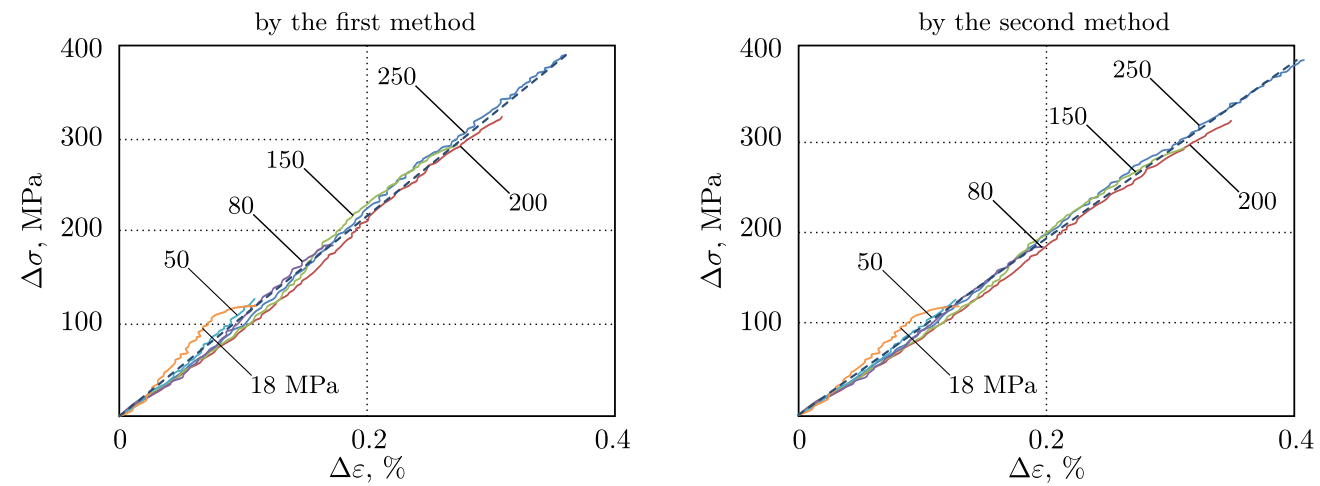

Рис. 8. (онлайн в цвете) Зависимость приращения объемной деформации $(\Delta \varepsilon)$ от приращения среднего давления $(\Delta \sigma)$ для перидотита: штриховая линия - теоретическая зависимость, сплошные линии - зависимости для боковых давлений, когда упругие константы определены по первому способу (слева) и по второму способу (справа)

[Figure 8. (color online) Plots of dependence between a volume deformation increment $(\Delta \varepsilon)$ and a mean pressure $(\Delta \sigma)$ for Nabe-ishi Peridotite: dashed line is theoretical dependence, solid lines are dependencies for lateral pressures when the elastic constants are defined by the first method (left) and by the second method (right)] 
тельный выбор того или иного метода определения упругих констант зависит от наличия экспериментальных данных при разных траекториях сложного нагружения.

\section{5. Отображение полной картины упругой деформации на всем} участке рассматриваемой траектории сложного нагружения. В качестве примера рассмотрим полученные данные для диорита. Задавая для него модуль Юнга $E=40660$ МПа и коэффициент Пуассона $\nu=0.23$, определенные по второму способу, восстановим полную картину осевой деформации для всех осуществленных траекторий сложного нагружения начиная с деформирования из исходного состояния и вплоть до соответствующих уровней напряжения $\sigma_{1}$, достигаемых при заданном значении $c$. Экспериментально диаграмма такой полной деформации в указанных пределах получается следующим образом. При выбранном виде напряженного состояния $(c)$ экспериментальную зависимость $\Delta \varepsilon_{1}\left(\Delta \sigma_{1}\right)$ дополним расчетным значением $\Delta \varepsilon_{1}^{0}\left(P_{H}\right)$ в области начального гидростатического давления, используя зависимость (10): при $\Delta \sigma_{1} \in\left[0, P_{H}\right]$ имеем $c=1$, т.е. согласно (10)

$$
\Delta \varepsilon_{1}^{0}=\frac{\Delta \sigma_{1}}{E}(1-2 \nu) \quad\left(0 \leqslant \Delta \sigma_{1} \leqslant P_{H}\right) .
$$

Соответствующая расчетная зависимость $\varepsilon_{1 \text { calc }}\left(\sigma_{1}\right)$ получается при использовании формулы (1). Наблюдаемое в опыте приращение $\Delta \varepsilon_{1}\left(\Delta \sigma_{1}\right)$ дополняется прогнозируемым, согласно $(11)$, приращением $\Delta \varepsilon_{1}^{0}\left(P_{H}\right)$ :

$$
\varepsilon_{1 \exp }\left(\sigma_{1}\right)=\Delta \varepsilon_{1}^{0}\left(P_{H}\right)+\Delta \varepsilon_{1}\left(\Delta \sigma_{1}\right) .
$$

В качестве примера сравним получаемые таким образом теоретические и «полуэмпирические» значения деформации $\varepsilon_{1}\left(\sigma_{1}\right)$ при двух видах напряженного состояния $(c=0.29, c=0.49)$. Результаты сравнения этих величин деформации (суммарной за всю историю нагружения) $\varepsilon_{1}$ представлены в табл. 3 (здесь $\left.\Delta \tilde{\varepsilon}_{1}=\frac{\varepsilon_{1 \text { calc }}-\varepsilon_{1 e x p}}{\varepsilon_{1 \text { exp }}} \cdot 100 \%\right)$ для таких уровней напряжения $\sigma_{1}$, которые вычисляются при заданных значениях $c$ и реализованных в опыте боковых давлениях. Наибольшее расхождение между расчетным и «полуэмпирическим» значениями деформации $\varepsilon_{1}$ получилось при боковом давлении $P_{H}=18$ МПа. Это объясняется наличием на исходной экспериментальной диаграмме $\Delta \varepsilon_{1} \sim \Delta \sigma_{1}$ начального нелинейного участка, который обычно наблюдается для подобных пород при малых уровнях напряжений [19].

6. Итоговое сопоставление расчетных и представленных в [1] экспериментальных диаграмм. Приращения осевой упругой деформации на втором звене рассматриваемой траектории сложного нагружения при разных боковых давлениях вычисляются по формуле (2). Результат сравнения расчетных диаграмм $\Delta \varepsilon_{1} \sim \Delta \sigma_{1}$ с экспериментальными данными представлен на рис. 9 и 10; при этом использованы значения упругих констант, найденные по первому и второму способу их определения. 

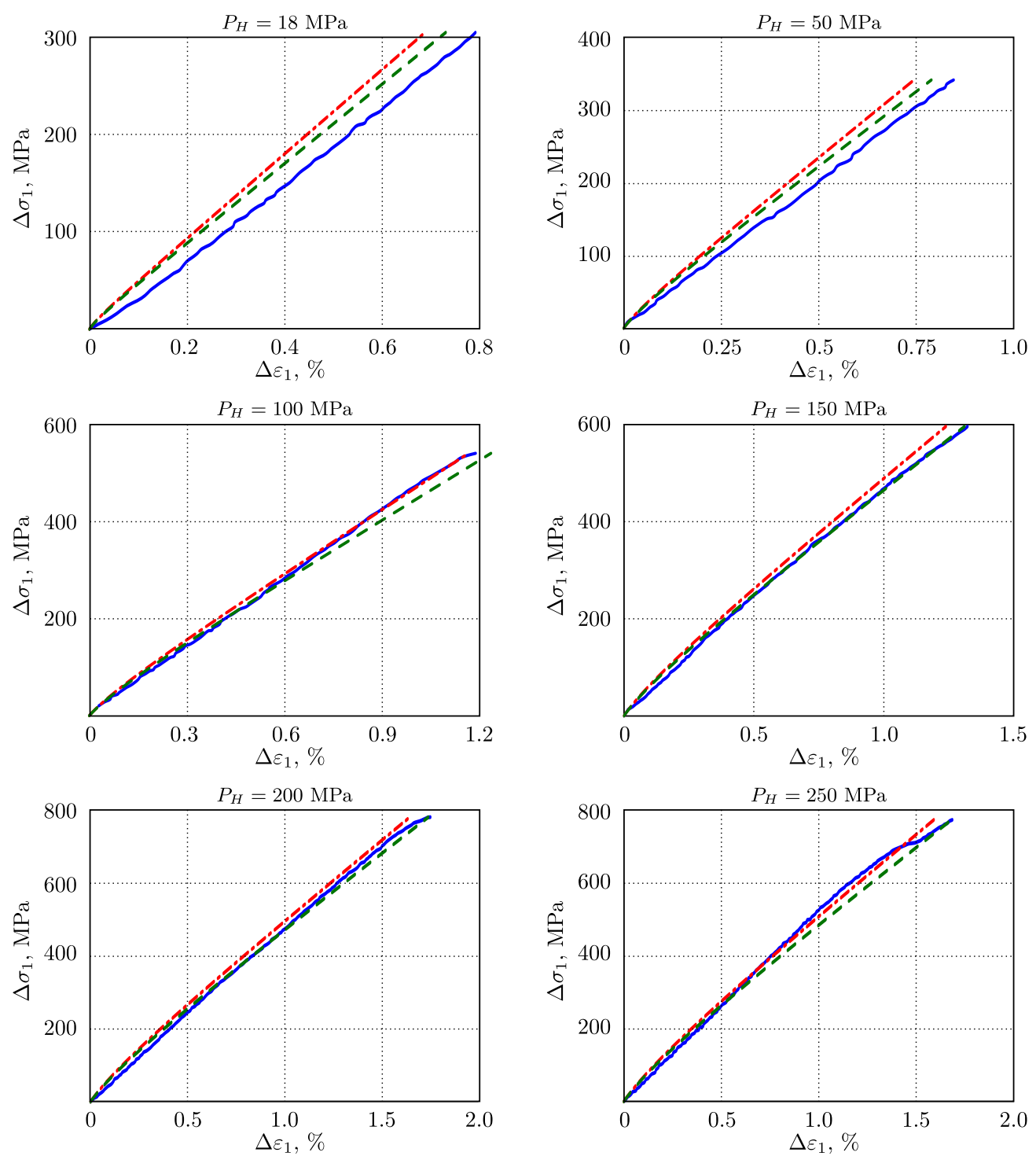

Рис. 9. Зависимости $\Delta \varepsilon_{1}\left(\Delta \sigma_{1}\right)$ для диорита при различных боковых давлениях

[Figure 9. Plots of dependence between $\Delta \varepsilon_{1}$ and $\Delta \sigma_{1}$ for Orikabe Diorite at various lateral pressures] 

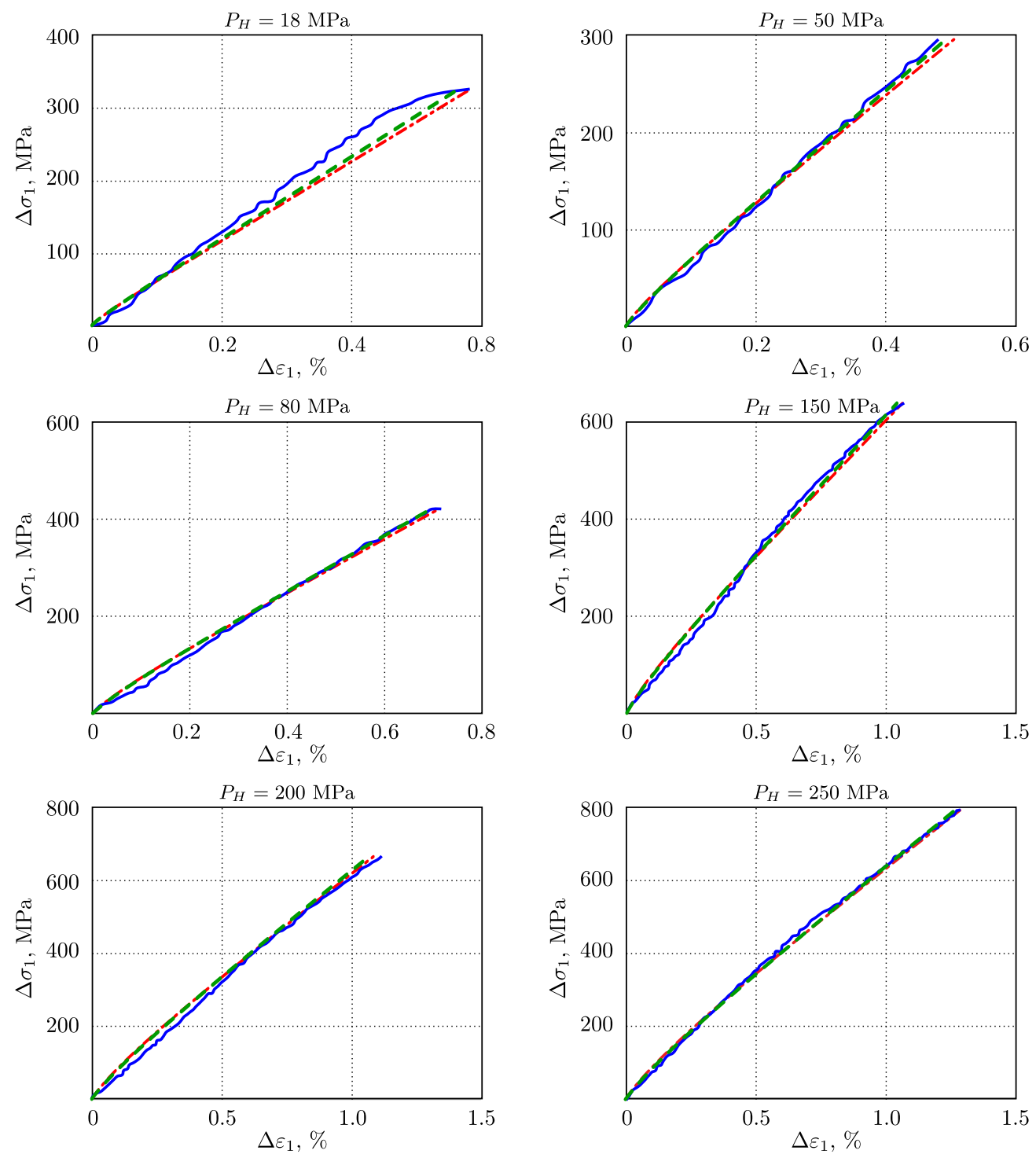

experimental data

by the first method $\quad-\quad---$ by the second method

Рис. 10. Зависимости $\Delta \varepsilon_{1}\left(\Delta \sigma_{1}\right)$ для перидотита при различных боковых давлениях

[Figure 10. Plots of dependence between $\Delta \varepsilon_{1}$ and $\Delta \sigma_{1}$ for Nabe-ishi Peridotite at various lateral pressures] 
Таблица 3 Сравнение теоретических и «полуэмпирических» значений деформации $\varepsilon_{1}\left(\sigma_{1}\right)$ при фиксированном виде напряженного состояния для диорита [Comparison of the theoretical and "semi-emperical" deformation values $\varepsilon_{1}\left(\sigma_{1}\right)$ for a fixed type of the stress state for Orikabe Diorite]

\begin{tabular}{|c|c|c|c|c|}
\hline$P_{H}, \mathrm{MPa}$ & $\sigma_{1}, \mathrm{MPa}$ & $\varepsilon_{1 e x p}, \%$ & $\varepsilon_{1 \text { calc }}, \%$ & $\Delta \tilde{\varepsilon}_{1}, \%$ \\
\hline \multicolumn{5}{|c|}{ for $c=0.29$} \\
\hline 250 & 861.63 & 1.522622 & 1.836427 & 20.610 \\
\hline 200 & 689.54 & 1.298017 & 1.469636 & 13.222 \\
\hline 150 & 519.32 & 0.986013 & 1.107437 & 12.315 \\
\hline 100 & 345.33 & 0.660409 & 0.736003 & 11.447 \\
\hline 50 & 172.47 & 0.363655 & 0.367598 & 1.084 \\
\hline 18 & 61.99 & 0.159406 & 0.132122 & -17.116 \\
\hline \multicolumn{5}{|c|}{ for $c=0.49$} \\
\hline 250 & 510.47 & 0.831022 & 0.972474 & 17.022 \\
\hline 200 & 408.14 & 0.693217 & 0.777536 & 12.163 \\
\hline 150 & 308.66 & 0.512912 & 0.588020 & 14.643 \\
\hline 100 & 204.00 & 0.347209 & 0.388624 & 11.928 \\
\hline 50 & 102.00 & 0.186304 & 0.194316 & 4.300 \\
\hline 18 & 37.13 & 0.088216 & 0,070730 & -19.822 \\
\hline
\end{tabular}

Заключение. Таким образом, как при пропорциональном нагружении [3], так и при сложном нагружении упругая деформация горных пород (изначально изотропных в исходном состоянии) характеризуется модулем Юнга и коэффициентом Пуассона как действительными константами материала. Как показало настоящее исследование, эмпирическая величина функции $E_{f}(c)$ (трактуемая как переменный упругий модуль [1]) согласуется с полученными в данном исследовании расчетными ее значениями в пределах естественного разброса экспериментальных данных.

В этой связи предлагаемое уточнение методики определения упругих констант при сложном нагружении необходимо как для практических расчетов в пределах упругости, так и для последующего правильного отображения [20] деформационного упрочнения горных пород.

Конкурирующие интересы. Конкурирующих интересов не имеем.

Авторский вклад и ответственность. Все авторы принимали участие в разработке концепции статьи и в написании рукописи. Авторы несут полную ответственность за предоставление окончательной рукописи в печать. Окончательная версия рукописи была одобрена всеми авторами.

Финансирование. Исследование выполнялось без финансирования.

\section{Библиографический список}

1. Mogi K. Experimental rock mechanics. London: CRC Press, 2007. 375 pp. doi: 10.1201/ 9780203964446.

2. Al-Shayea N. A. Effects of testing methods and conditions on the elastic properties of limestone rock// Engineering Geology, 2004. vol.74, no.1-2. pp. 139-156. doi: 10.1016/j. 
enggeo.2004.03.007.

3. Xu H., Zhou W., Xie R., et al. Characterization of Rock Mechanical Properties Using Lab Tests and Numerical Interpretation Model of Well Logs // Mathematical Problems in Engineering, 2016. vol.2016, no. 1, 5967159. 13 pp. doi : 10.1007/978-3-642-33911-0_1.

4. Стефанов Ю. П. Некоторые нелинейные эффекты поведения горных пород // Физическая мезомеханика, 2016. Т. 19, №6. С. 54-61.

5. Сукнёв С. В., Фёдоров С. П. Методы определения упругих свойств горных пород// Наука и образование, 2014. № 1(73). С. 18-24.

6. ГОСТ 28985-97. Породъ горнъе. Метод определения дебормационных характеристик при одноосном сжатии. М.: ИПК Издательство стандартов, 2004. 10 с.

7. DIN EN 14580:2005-07. Prüfverfahren für Naturstein - Bestimmung des statischen Elastizitätsmoduls [Natural stone test methods - Determination of static elastic modulus]. Berlin: Deutsches Institut für Normung, 2005. 15 pp. (In German)

8. ASTM D7012-10. Standard Test Methods for Compressive Strength and Elastic Moduli of Intact Rock Core Specimens under Varying States of Stress and Temperatures. West Conshohocken, PA: ASTM international, 2010. doi: 10.1520/D7012-10.

9. Franklin J. A., Hoek E. Developments in Triaxial Testing Equipment // Rock Mech., 1970. vol. 2. pp. 223-228.

10. Ma C.; Guo C.; Pan S., Zhou Y. Development and application of rock triaxial tensile test device// Rock and Soil Mechanics. vol.39, no. 1. pp. 537-543. doi: 10.16285/j.rsm.2017. 2541.

11. Bukowska M., Sanetra U. The tests of the conventional triaxial granite and dolomite compression in the aspect of their mechanical properties// Mineral Resources Management, 2008. vol. 24, no. 2. pp. 345-358.

12. Nowakowski A. On certain determinantal method of equation and effective pressure evaluation on the basis of laboratory researches // Archives of Mining Sciences, 2007. vol.52, no. 4. pp. $587-610$.

13. Kamp L. T., Konietzky H., Blumling P., et al. Micromechanical back-analysis of laboratory tests on rock / Numerical Models in Geomechanics - NUMOG VII, 1999. pp. 411-416.

14. Сукнёв С. В. Определение модуля упругости горных пород при сжатии // Заводская лаборатория. Диагностика материалов, 2017. Т. 83, № 12. С. 52-57. doi: 10.26896/ 1028-6861-2017-83-12-52-57.

15. Paterson M. S., Wong T. Experimental Rock Deformation - The Brittle Field. Berlin: Springer, 2005. x+347 pp. doi : 10.1007/b137431.

16. Mishra D. A., Janeček I. Laboratory Triaxial Testing — from Historical Outlooks to Technical Aspects // Procedia Engineering, 2017. vol. 191. pp. 342-351. doi : 10.1016/j .proeng. 2017.05 .190 .

17. Li X., Shi L., Bai B., Li Q., Xu D., et al. True-triaxial testing techniques for rocks-State of the art and future perspectives / True triaxial testing of rocks / Geomechanics Research Series, 4. Leiden, Netherlands: CRC Press, 2012. pp. 3-18.

18. Михайлов-Михеев П. Б. Справочник по металлическим материалам турбино- и мотостроения. М.-Л.: Машгиз, 1961. 839 с.

19. Ставрогин А. Н., Протосеня А. Г. Пластичность горнъх пород. М.: Недра, 1979.305 с.

20. Рычков Б. А. О деформационном упрочнении горных пород// Изв. PAH. MTT, 1999. № 2. С. 115-124. 


\title{
On the interpretation of rocks elasticity modulus
}

\author{
N. M. Komartsov, M. A. Kulagina, B. A. Rychkov
}

Kyrgyz-Russian Slavic University named after B. N. Eltsin, 44, Kievskaya st., Bishkek, 720000 Kyrgyz Republic.

\begin{abstract}
We consider the experimental data of the testing of rocks, performed by $K$. Mogi in his monograph "Experimental rock mechanics" which was published in 2007. Cylindrical samples of rocks were tested according to T. Karman's scheme: on the first step, hydrostatic pressure was created up to different stress levels, on the second step the axial load was increased at a constant level of reached lateral pressure. During such a complex loading on the second step only the increment of axial strain was measured, which depends of the increment of the axial stress. In origin this dependence is presented in the form of graphs in real scale, which made it possible to convert these graphs into digital format in the form of tabular values. Two rocks are considered: Orikabe Diorite and Nabe-ishi Peridotite.

According to the tables obtained, the stress-deformed state of these rocks in the second stage of complex loading for six test programs carried out in the experiment was analyzed. On each implemented loading path, a point is selected that corresponds to the axial stress with the same form of stress state. The latter, as is customary in geomechanics, is characterized by the ratio of the average principal stress to the maximum main stress. Thus, a (calculated) trajectory of proportional loading is distinguished for all stress levels within the elastic range. It is demonstrated that for such calculated loading paths, the experimental value of the increment of axial strain (within the elastic range) is a linear function of the increment of the axial stress. This proves the applicability of the generalized Hooke's law.

As a result, the Young's modulus and the Poisson's ratio are determined and shown that they (with respect to rocks) are indeed elastic constants,
\end{abstract}

\section{Research Article}

๑ (ㅇ) The content is published under the terms of the Creative Commons Attribution 4.0 International License (http://creativecommons.org/licenses/by/4.0/)

Please cite this article in press as:

Komartsov N. M., Kulag in a M. A., Ry chkov B. A. On the interpretation of rocks elasticity modulus, Vestn. Samar. Gos. Tekhn. Univ., Ser. Fiz.-Mat. Nauki [J. Samara State Tech. Univ., Ser. Phys. Math. Sci.], 2018, vol. 22, no. 3, pp. 487-503. doi: 10.14498/vsgtu1604 (In Russian).

\section{Authors' Details:}

Nikita M. Komartsov (D) http://orcid.org/0000-0003-0266-6271

Cand. Phys. \& Math. Sci., Associate Professor; e-mail: komartsovnm@mail.ru

Margarita A. Kulagina (D) http://orcid.org/0000-0001-7979-7881

Postgraduate Student; e-mail: kulagina_m.a@mail.ru

Boris A. Rychkov (10) http://orcid.org/0000-0001-7408-1657

Dr. Phys. \& Math. Sci., Professor; e-mail: rychkovba@mail.ru 
and not variable quantities, as it is sometimes interpreted.

Keywords: elastic deformation of rocks, elastic constants, applicability of Hooke's law under complex loading.

Received: $30^{\text {th }}$ January, $2018 /$ Revised: $17^{\text {th }}$ August, $2018 /$

Accepted: $3^{\text {rd }}$ September, $2018 /$ First online: $9^{\text {th }}$ October, 2018

Competing interests. We have no competing interests.

Authors' contributions and responsibilities. Each author has participated in the article concept development and in the manuscript writing. The authors are absolutely responsible for submitting the final manuscript in print. Each author has approved the final version of manuscript.

Funding. The research has not had any funding.

\section{References}

1. Mogi K. Experimental rock mechanics. London, CRC Press, 2007, 375 pp. doi: 10.1201/ 9780203964446.

2. Al-Shayea N. A. Effects of testing methods and conditions on the elastic properties of limestone rock, Engineering Geology, 2004, vol.74, no.1-2, pp. 139-156. doi: 10.1016/j . enggeo.2004.03.007.

3. Xu H., Zhou W., Xie R., et al. Characterization of Rock Mechanical Properties Using Lab Tests and Numerical Interpretation Model of Well Logs, Mathematical Problems in Engineering, 2016, vol. 2016, no. 1, 5967159, 13 pp. doi : 10.1007/978-3-642-33911-0_1.

4. Stefanov Yu. P. Some nonlinear rock behavior effects, Physical Mesomechanics, 2018, vol. 21, no. 3, pp. 234-241. doi: 10.1134/S1029959918030074.

5. Suknev S. V., Fedorov S. P. Methods of determination of rock elastic properties, Nauka $i$ obrazovanie, 2014, no. 1(73), pp. 18-24 (In Russian).

6. GOST 28985-97. Rocks. Method of determination of deformation characteristics under uniaxial compression. Moscow, Izdatel'stvo Standartov, 2004, 10 pp. (In Russian)

7. DIN EN 14580:2005-07. Prüfverfahren für Naturstein - Bestimmung des statischen Elastizitätsmoduls [Natural stone test methods - Determination of static elastic modulus]. Berlin, Deutsches Institut für Normung, 2005, 15 pp. (In German)

8. ASTM D7012-10. Standard Test Methods for Compressive Strength and Elastic Moduli of Intact Rock Core Specimens under Varying States of Stress and Temperatures. West Conshohocken, PA, ASTM international, 2010. doi: 10.1520/D7012-10.

9. Franklin J. A., Hoek E. Developments in Triaxial Testing Equipment, Rock Mech., 1970, vol. 2, pp. 223-228.

10. Ma C.; Guo C.; Pan S., Zhou Y. Development and application of rock triaxial tensile test device, Rock and Soil Mechanics, vol.39, no.1, pp. 537-543. doi:10.16285/j.rsm.2017. 2541.

11. Bukowska M., Sanetra U. The tests of the conventional triaxial granite and dolomite compression in the aspect of their mechanical properties, Mineral Resources Management, 2008, vol. 24, no. 2, pp. 345-358.

12. Nowakowski A. On certain determinantal method of equation and effective pressure evaluation on the basis of laboratory researches, Archives of Mining Sciences, 2007, vol.52, no. 4, pp. 587-610.

13. Kamp L. T., Konietzky H., Blumling P., et al. Micromechanical back-analysis of laboratory tests on rock, In: Numerical Models in Geomechanics - NUMOG VII, 1999, pp. 411-416. 
14. Suknev S. V. Determination of the modulus of elasticity of rocks upon compression, Industrial Laboratory. Diagnostics of Materials, 2017, vol. 83, no. 12, pp. 52-57 (In Russian). doi : 10.26896/1028-6861-2017-83-12-52-57.

15. Paterson M. S., Wong T. Experimental Rock Deformation - The Brittle Field. Berlin, Springer, 2005, $\mathrm{x}+347$ pp. doi: $10.1007 / \mathrm{b} 137431$.

16. Mishra D. A., Janeček I. Laboratory Triaxial Testing - from Historical Outlooks to Technical Aspects, Procedia Engineering, 2017, vol.191, pp. 342-351. doi:10.1016/j.proeng. 2017.05 .190$.

17. Li X., Shi L., Bai B., Li Q., Xu D., et al. True-triaxial testing techniques for rocks-State of the art and future perspectives, In: True triaxial testing of rocks, Geomechanics Research Series, 4. Leiden, Netherlands, CRC Press, 2012, pp. 3-18.

18. Mikhailov-Mikheev P. B. Spravochnik po metallicheskim materialam turbino- $i$ motostroeniia [Handbook of Metallic Materials of Turbine and Motor Industry]. Moscow, Leningrad, Mashgiz, 1961, 839 pp. (In Russian)

19. Stavrogin A. N., Protosenia A. G. Plastichnost' gornykh porod [Plasticity of rocks]. Moscow, Nedra, 1979, 305 pp. (In Russian)

20. Rychkov B. A. On strain hardening of rock materials, Mechanics of Solids, 1999, vol. 34, no. 2, pp. 97-104. 